\title{
Comparison of two acoustic analogies applied to experimental PIV data for cavity sound emission estimation
}

\author{
Valentina Koschatzky, Jerry Westerweel, Bendiks Jan Boersma \\ Delft University of Technology, Delft, Mekelwel 2, 2628 CD, The Netherlands
}

\begin{abstract}
The aim of the present study is to compare two different acoustic analogies applied to time-resolved particle image velocimetry (PIV) data for the prediction of the acoustic far-field generated by the flow over a rectangular cavity. Recent developments in laser and camera technology allow the possibility to extend PIV to the study of aeroacoustic phenomena in air flows at moderate speed (up to $\mathrm{V}=20 \mathrm{~m} / \mathrm{s}, \mathrm{Ma}=0.08$ ). We make use of these new possibilities to obtain estimates of the acoustic emission from time-resolved PIV data. We consider the model problem of the sound radiating from an open, two dimensional, shallow cavity with an aspect ratio between its length and depth of 2 at a Reynolds number of $3.0 \times 10^{4}$ based on the cavity length. The study is carried out combining high speed two-dimensional PIV imaging and sound measurements. The emitted sound is then calculated using Curle's analogy and vortex sound theory. The prediction of the acoustic fields obtained by applying the two methods are analyzed and compared with the measured sound. Results show that both the analogies estimate the overall sound pressure level quite well and within a few $\mathrm{dB}$ of each other. Vortex sound theory seems to provide a better estimate for the amplitude of the tonal component and its harmonics, but suffers from higher broadband noise compare to Curle's analogy. This is due to the higher smoothing of the data involved in the computation of the source term in Curle's analogy, which reduces noise but also smoothes out the signal. Results show that great care must be taken in the treatment of the experimental PIV data, especially in the techniques used for spatial and temporal differentiation. The data sets obtained by PIV measurements can be, in fact, quite noisy while the spatial and temporal resolution are still limited.
\end{abstract}

\section{Introduction}

As a test case for the comparison of the application of two acoustic analogies to experimental PIV data, we chose to study the flow over a rectangular open ${ }^{\mathrm{a}}$ cavity. This geometry is well suited for the development and testing of a new experimental technique for the study of aeroacoustics of wall-bounded flows. It generates a strong periodic acoustic emission with a large amplitude. We give here a brief description of the flow features and a summary of the research done on the subject. The generation of noise by flow over a rectangular cavity is an important benchmark problem for aeroacoustics, and it has been investigated both experimentally and numerically in the last decades. ${ }^{1}$ Ahuja and Mendoza ${ }^{2}$ have shown how the flow that generates over open cavities depends on multiple factors, such as the cavity geometry, the free stream velocity and properties of the upstream boundary layer. Gharib and Roshko ${ }^{3}$ identified different flow behavior arising in flows over rectangular open cavities. In particular, they showed that, when the ratio between the cavity length and the boundary layer momentum thickness falls within certain values $(80 \div 100<\mathrm{L} / \theta>120 \div 140$ depending also on other parameters), the flow passing over the cavity develops in what is known as the "shear layer mode": the shear layer separating at the leading edge of the cavity develops into largescale coherent spanwise vortices. These vortical structures periodically impinge on the aft wall of the cavity, producing pressure fluctuations that radiate acoustic waves and that generate a self-sustaining oscillation mechanism. This mechanism has a double nature: both acoustic and hydrodynamic. Which one predominates depends on the wavelength of the perturbation, and therefore on the flow speed and the characteristic dimension of the cavity, i.e. the streamwise length of the cavity. In low speed flows, the cavity can be considered as acoustically compact: its length is much smaller than the acoustic wavelength. Therefore, the acoustic perturbation is not able to influence the flow behavior in the cavity, and hence the self-sustaining oscillation mechanism is purely hydrodynamic. It consists of a recirculating

\footnotetext{
${ }^{*} \mathrm{PhD}$ student, Laboratory for Aero and Hydrodynamics, Leeghwaterstraat 21, $2628 \mathrm{CA}$ Delft.

${ }^{\dagger}$ Professor, Laboratory for Aero and Hydrodynamics, Leeghwaterstraat 21, 2628 CA Delft.

¥Professor, Laboratory for Aero and Hydrodynamics, Leeghwaterstraat 21, 2628 CA Delft.

${ }^{a} \mathrm{~A}$ cavity is called open when the ratio between its length and depth is larger than one
} 
vortex inside the cavity that impinges on the shear layer, enhancing and triggering its de-stabilization. The compactness assumption is fundamental in the use acoustic analogies for the prediction of the sound emission since it allows us to decouple the acoustic features of the flow from the hydrodynamic ones, i.e. to have to solve an explicit equation. Experimental studies have usually been confined to single-point time-resolved measurements or full-field PIV imaging at low speeds. ${ }^{2,4-6}$ Some of the more recent attempts, such as that of Haigermoser et al. ${ }^{7}$ and Haigermoser, ${ }^{8}$ have performed time-resolved PIV measurements, in water flows and at low free-stream velocity $\left(R e=7.8 \times 10^{3}\right)$. This is a noticeable step forward, but at conditions that are not yet representative of significant acoustic noise generation. In previous studies ${ }^{9-11}$ we focused on the application of Curle's analogy to compute the acoustic pressure from the PIV data. Here we present a different approach by estimating the emitted sound by using the theory of vortex sound derived by Powell ${ }^{12}$ and developed by Howe. ${ }^{13}$

\section{Description of the experiment}

The study was carried out combining high speed planar PIV imaging and sound measurements. We performed measurements with a laminar boundary layer flow approaching the cavity, at a free-stream velocity of $15 \mathrm{~m} / \mathrm{s}$, giving a Reynolds number of $3.0 \times 10^{4}$ based on the cavity length, $L$. For the investigated flow condition, the ratio between the length of the cavity and the boundary layer momentum thickness (determined from the velocity profile that was measured by traversing an hot wire anemometer) was $L / \theta=112$. At this condition the flow passing over the cavity induces the "shear layer mode" described in the introduction. Measurements were conducted in the vertical open-jet wind tunnel of the Low-speed Aerodynamics Laboratories of the Aerospace Department at the Delft University of Technology. The cavity was machined in a flat plate and positioned in the test section aligned with the flow. Figure 1 shows a schematic representation of the experimental configuration including the dimensions of the cavity. The cavity had a width of $600 \mathrm{~mm}$ spanning the full width of the tunnel and giving a width to length ratio of $W / L=20$. Details of the measurements and the experimental conditions are given elsewhere. ${ }^{11}$
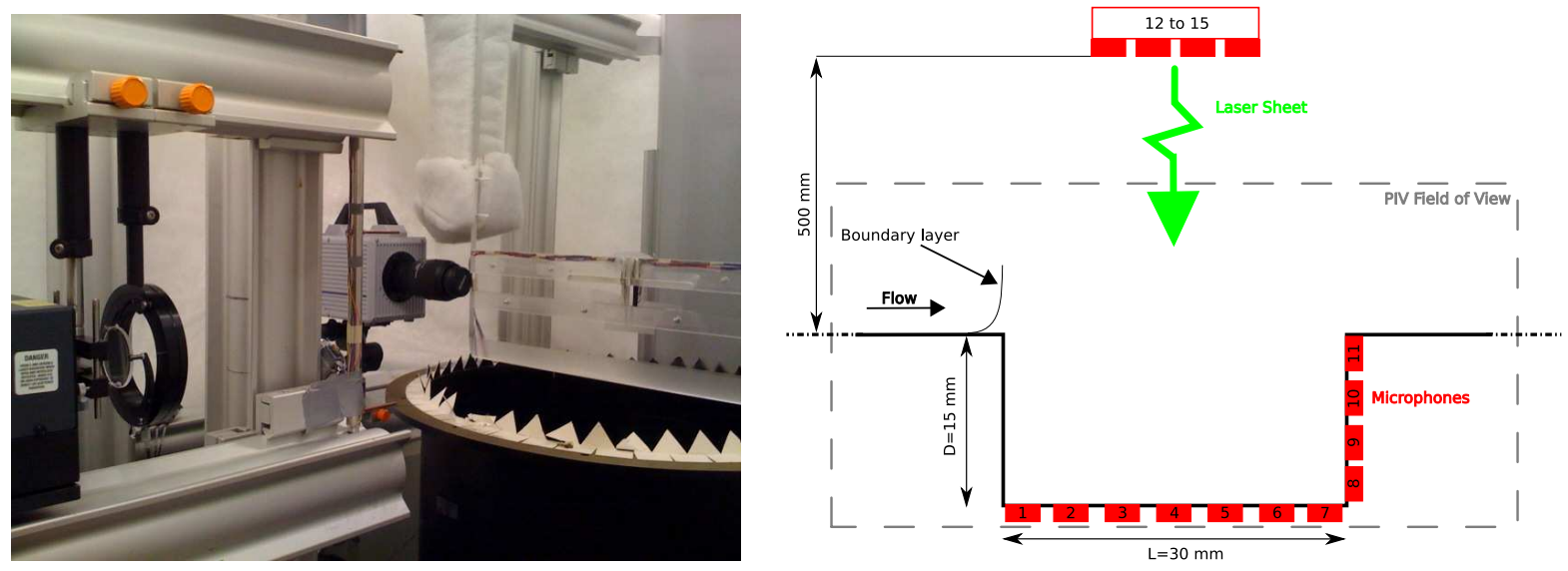

Figure 1. The experimental setup.

\section{A. PIV setup}

The PIV system consisted of a 12-bit 1024x1024 pixels camera(Photron FastCAM A1S), used at a image format of $512 \times 1024$ pixels to allow an increase of the frame rate that matches the minimum required, and a dual-cavity pulsed Nd:YLF laser (New Wave Pegasus). The flow was seeded by means of a stage smoke generator (Safex) that produces particles of approximately $1 \mu \mathrm{m}$ in diameter. PIV image pairs were acquired at a frequency of $3 \mathrm{kHz}$ with a time delay of $65 \mu$ s between the first and second frame. This time delays was chosen as an optimum between two opposing demands, i.e. allowing enough particle-image displacement between the two frames in the slower regions of our flow inside the cavity (in the order of 2 to 3 pixels), while keeping it within reasonable values in the regions with a faster flow (typically 16 pixels in the free-stream area). The laser sheet was estimated to be approximately $1 \mathrm{~mm}$ thick, which was determined by projection onto a millimetric scale. This is comparable to the in-plane dimension of our final interrogation window. Data were processed using a multi-pass algorithm, ${ }^{14}$ with the interrogation window sizes tailored to the local flow regime. This approach was chosen to deal with the large dynamic range of the flow. 


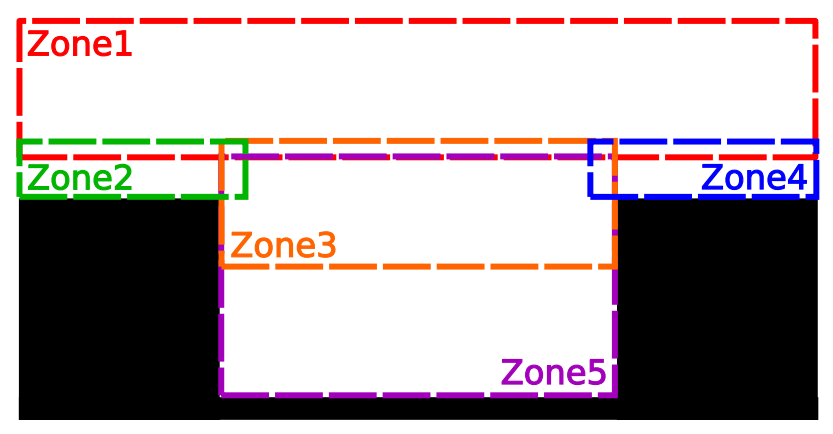

\begin{tabular}{|l|l|l|l|l|}
\hline \multicolumn{1}{|c|}{ Zone } & Pass 1 & Pass 2 & Pass 3 & Pass 4 \\
\hline \hline 1: Free flow & $\begin{array}{l}32 \times 32 \\
\text { ps }\end{array}$ & $16 \times 16$ & $16 \times 16$ & none \\
\hline $\begin{array}{l}\text { 2: } \text { Incoming } \\
\text { boundary layer }\end{array}$ & $64 \times 8$ & $32 \times 4$ & $16 \times 4$ & $16 \times 4$ \\
\hline 3: Shear layer & $\begin{array}{l}64 \times 64 \\
\text { wd }\end{array}$ & $\begin{array}{l}32 \times 32 \\
\text { wd }\end{array}$ & $\begin{array}{l}16 \times 16 \\
\text { wd }\end{array}$ & $\begin{array}{l}16 \times 16 \\
\text { wd }\end{array}$ \\
\hline $\begin{array}{l}\text { 4: } \text { After the cav- } \\
\text { ity }\end{array}$ & $\begin{array}{l}64 \times 32 \\
\text { wd }\end{array}$ & $\begin{array}{l}32 \times 16 \\
\text { wd }\end{array}$ & $\begin{array}{l}16 \times 16 \\
\text { wd }\end{array}$ & $\begin{array}{l}16 \times 16 \\
\text { wd }\end{array}$ \\
\hline $\begin{array}{l}\text { 5: } \text { Inside the cav- } \\
\text { ity }\end{array}$ & $\begin{array}{l}32 \times 32 \\
\text { wd }\end{array}$ & $\begin{array}{l}16 \times 16 \\
\text { wd }\end{array}$ & $\begin{array}{l}16 \times 16 \\
\text { wd }\end{array}$ & none \\
\hline
\end{tabular}

Figure 2. Interrogation settings in the various flow domains. Everywhere a $50 \%$ overlap between adjacent interrogation domains was used a 50\%. ps: pre shift, wd: window deformation

Figure 2 shows how the measurement domain was divided in the different areas and it contains a summary of the initial and intermediate dimensions and settings of the interrogation domains. The upstream boundary layer region was particularly sensitive to the processing parameters, because of the strong gradients in the wall-normal direction. The interrogation windows were placed in such a way that their boundaries coincided with the walls of the cavity. The distance between the cavity walls and the location of the first vector is therefore half the size of a window. The final passes in all domains were done with square interrogation windows of $16 \times 16$ pixels with a $50 \%$ overlap, giving a vector spacing of $0.48 \mathrm{~mm}$ throughout the entire measurement domain.

\section{B. Sound measurements}

The emitted sound was recorded with 4 microphones (Sonion serie8000) positioned 0.5 meters above the cavity lid plane. The sound pressure was acquired at a frequency of $100 \mathrm{kHz}$ during the acquisition of the PIV data. The analog microphone signals where amplified in order to get an optimum signal dynamic range. The microphones were then calibrated a posteriori by comparison with a calibrated microphone (CESVA P-05) that was exposed together with the other microphones used in the measurement (including the analog signal amplifiers) to the same acoustic field. This field was generated by a signal generator unit and a loudspeaker. We performed measurements for a wide range of amplitudes and frequencies. As specified by the manufacturer of the microphones, the amplitude response appeared to be independent of the frequency within our measurement range (from $100 \mathrm{~Hz}$ and to $8 \mathrm{kHz}$ ). The conversion from electric signal (in Volts) to pressure (in Pa) appeared to be given by a linear relation (with a linear regression coefficient, $R^{2}$, that is better than 0.99) and with a zero offset, i.e. the conversion reduces to a single gain coefficient.

For further details about the experimental setup we refer to the paper by Koschatzky et al. ${ }^{11}$

\section{Acoustic analogies}

The emitted sound is computed using both Curle's analogy and vortex sound theory. Both analogies are derived from the compressible Navier-Stokes equations rearranged into two different forms of an inhomogeneous wave equation. The compactness assumption allows us to decouple the acoustic features of the flow from the hydrodynamic ones, so the acoustic variable and the source term in the wave equation can be considered as independent, and the problem becomes explicit. In both analogies we took into account the presence of the non-compact wall, in which the cavity is present, by using the image principle in the derivation of the solution. Figure 3 summarizes the coordinate system and notation used in the analogies: $\mathbf{y}$ is the source location, $\mathbf{x}$ is the listener location, and $\overline{\mathbf{x}}=\left(\begin{array}{lll}x_{1}, & -x_{2}, & x_{3}\end{array}\right)$ is its image on the opposite side of the wall. Subscripts $i=1,2$ and 3 refers to the streamwise, normal and spanwise directions, respectively. The assumption of compactness implies that $|\mathbf{x}| \rightarrow \infty$ and $|\mathbf{x}| \gg|\mathbf{y}|$.

\section{A. Curle's Analogy}

The first approach we consider for the computation of the acoustic pressure from the PIV data is to apply Curle's analogy. ${ }^{15}$ Curle's acoustic analogy is derived from Lighthill's analogy, ${ }^{16}$ where the chosen acoustic variable is the perturbation density $\rho-\rho_{0}$. At low Mach number the perturbation pressure can be expressed as: $p-p_{0}=\left(\rho-\rho_{0}\right) a_{0}^{2}$, 


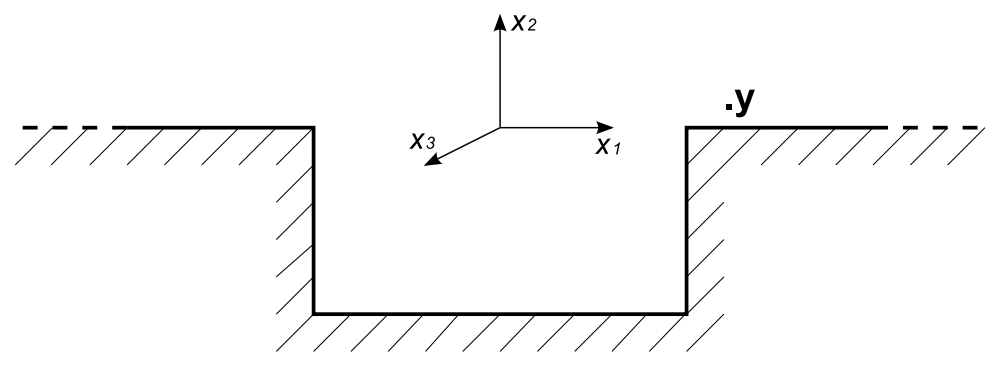

$\overline{\mathbf{x}}$

Figure 3. reference system notation used in this paper

where $a_{0}$ is the speed of sound. Under the same assumption, in high Reynolds numbers flows, and for listeners positioned in the far field, Curle's equation can be further simplified: the volume term can be ignored, as well as the viscous terms, and a compact form can be adopted. To take into account the presence of the non-compact wall in which the cavity take place, we follow the approach introduced by Powell: ${ }^{17}$ we apply Curle's solution both at the observer point $\mathbf{x}$ and at its image on the opposite side of the wall $\overline{\mathbf{x}}$, and we simply add the two solutions. It follows that the contribution to the sound emission from pressure fluctuations at walls with normal $n_{2}$ is null while the ones with normals $n_{1}$ and $n_{3}$ contribute with a double intensity that in the free space solution. That means that the walls before and after the cavity and the bottom of the cavity are "silent" and sound is produced only by pressure fluctuations at the vertical walls of the cavity. Curle's equation then takes the form:

$$
p(\mathbf{x}, t)-p_{0}=\frac{x_{i}}{2 \pi a_{0}|\mathbf{x}|^{2}} \int_{S}\left[\frac{\partial p}{\partial t}\right] n_{i} d S(\mathbf{y}), \quad i=1,3,
$$

where $p$ is the pressure, $n$ the wall normal pointing into the flow, and $a_{0}$ the speed of sound. The index $i$ is in principle either 1 or 3 , but in the present configuration there are no walls with a normal component in the direction with $i=3$, so the index to consider here is in practice just $i=1$. The term in the square brackets in (1) is evaluated at the retarded time, $t-|\mathbf{x}| / a_{0}$. Equation (1) relates the acoustic pressure at the listener position, $\mathbf{x}$, to a surface integral of the time derivative of the hydrodynamic wall pressure, evaluated at a retarded time that takes into account the distance between the source and the listener position. The main issue is then to compute the pressure and then its time derivative at the walls from the PIV data. In order to do so, we solve a Poisson equation for the pressure derived from the momentum equation in which we ignore the viscous terms, since we are considering a flow with a relatively high Reynolds for which the contribution of viscous terms is considered to be negligible; see Liu and Katz. ${ }^{18}$ To compute the time derivatives of the two velocity components in the momentum equation, we use the method proposed by Liu and Katz ${ }^{18}$ and recently developed by Moore et al. ${ }^{19}$ The method consists of the evaluation of the total derivative of the velocity by tracking a fluid parcel by means of a Lagrangian approach, under the assumption of Taylor's hypothesis of frozen turbulence. This is permitted, since the characteristic fluctuations of the flow are small with respect to the characteristic velocity of the mean recirculating flow in the cavity, and the larger structures that are responsible for the major fraction of the total sound emission convect without much deformation within the duration of our acquisition. This approach is necessary due to the relatively large time steps between PIV snapshots, which do not allow for a correct computation of time derivatives when done in the conventional Eulerian way. The pressure time derivative is computed in the same way. For further details on the computation of the pressure and of the pressure time derivative refer to Koschatzky et al. ${ }^{11}$

\section{B. Vortex Sound Theory}

Powell ${ }^{12}$ derived a different form of Lighthill's analogy by considering the total enthalpy, $B$, instead of the fluid density, $\rho$, as the acoustic variable. Howe ${ }^{13}$ generalized Powell theory to allow its application to wall bounded flows. At low Mach number and in the far field the acoustic pressure $p-p_{0}$ can be expressed in terms of the total enthalpy $B$ as 
$p-p_{0}=\rho_{0} B$. We can then solve the acoustic equation for the perturbation pressure by means of a Green's function in a similar way as done in the derivation of Curle's analogy. The main difference is that, while in the derivation of Curle's analogy the solution is found in the free space and the body with its boundaries are introduced successively, leading to a surface integral term, here the presence of the solid boundary is part of the wave equation solution itself, since we use a Green's function tailored to the cavity geometry. In the derivation of the tailored Green's function we take into account the presence of the non-compact wall in which the cavity is located: we do that by expanding the Green's function solution for a flat plate to first order in $\mathbf{y}$ near the cavity, i.e. we correct to dipole order. ${ }^{13}$ We then tailor the Green's function on the cavity geometry, introducing the Kirchoff vector, Y, for the body. The components $Y_{i}$ of the Kirchoff vector are the velocity potentials of an incompressible flow past the cavity, having unit speed in the $i$-th direction at large distances from the cavity. The solution of the inhomogeneous wave equation then takes the form:

$$
p(\mathbf{x}, t)-p_{0}=-\frac{\rho x_{i}}{2 \pi a_{0}|\mathbf{x}|^{2}} \int_{V}\left[\frac{\partial}{\partial t}\left((\omega \times \mathbf{u}) \cdot \nabla Y_{i}(\mathbf{y})\right)\right] d V(\mathbf{y}), \quad i=1,3 .
$$

In equation (2) $\rho$ is the fluid density, and $\omega \times \mathbf{u}$ the Lamb vector, i.e. the cross product of vorticity $\omega$ and velocity $\mathbf{u}$. As for Curle's analogy, $i$ is either 1 or 3 . But since $\left|\nabla Y_{3}(\mathbf{y})\right| \ll\left|\nabla Y_{1}(\mathbf{y})\right|$ we consider just $i=1$. $Y_{1}$ is found by conformal mapping of the cavity geometry on a half plane, ${ }^{20}$ where the solution for the velocity potential is straightforward. In particular it is found that:

$$
Y_{1}(\mathbf{y})=\mathfrak{R}\left(\frac{L k}{2 E(k)} \xi(\mathbf{y})\right) \quad \text { and } \quad \mathbf{y}=\frac{L}{2 E(k)} E(\xi, k) \text {. }
$$

In equation (3), $\mathbf{y}=y_{1}+i y_{2}$ and $\xi=\xi_{1}+i \xi_{2}$ (as in figure 4 ), and $\Re(\cdot)$ indicates the real part of the argument, $L$ is the cavity length, $k$ a parameter for the aspect ratio of the cavity, ${ }^{20} E(k)$ a complete elliptical integral of the second kind, and $E(\xi, k)$ an incomplete elliptical integral of the second kind. ${ }^{21}$ Figure 4 shows streamlines and equipotential lines obtained by conformal mapping of the cavity (plane $y$ ) into an half plane (plane $\xi$ ). The terms within the square brackets in (2) are evaluated at the retarded time, $t-|\mathbf{x}| / a_{0}$. Equation (2) relates the acoustic pressure at the listener position, $\mathbf{x}$, to a volume integral of the time derivative of the Lamb vector, evaluated at a retarded time that takes into account the distance between the source and the listener position. The problem then reduces to the computation of

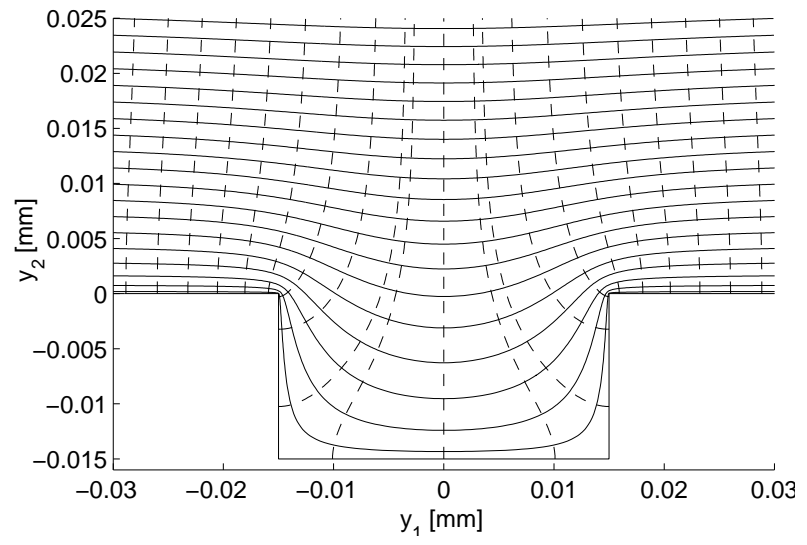

(a) Plane y

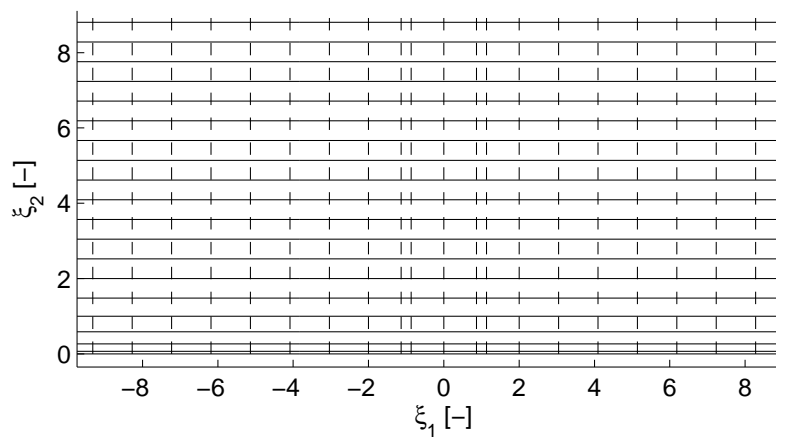

(b) Plane $\xi$

Figure 4. Streamlines (-) and equipotential lines (- -) in the $\mathrm{y}$ and $\xi$ planes.

the Lamb vector and its time derivative. The experimental data we use for the estimation of the emitted sound are planar, i.e. we dispose of the components $u_{1}$ and $u_{2}$ of the velocity field and of the component $\omega_{3}$ of the vorticity field. Therefore, some of the terms in the volume integral of (2) are missing. In particular, being:

$$
(\omega \times \mathbf{u}) \cdot \nabla Y_{1}=\left(\omega_{2} u_{3}-\omega_{3} u_{2}\right) \frac{\partial Y_{1}}{\partial y_{1}}+\left(\omega_{3} u_{1}-\omega_{1} u_{3}\right) \frac{\partial Y_{1}}{\partial y_{2}}+\left(\omega_{1} u_{2}-\omega_{2} u_{1}\right) \frac{\partial Y_{1}}{\partial y_{3}},
$$

and $\partial Y_{1} / \partial y_{3}=0$ (by definition), we lack the terms $\omega_{2} u_{3}$ and $\omega_{1} u_{3}$. Our flow is quasi two-dimensional, ${ }^{11}$ which implies $\left|\omega_{3}\right| \gg\left|\omega_{2}\right|,\left|\omega_{1}\right|$ and $\left|u_{3}\right| \ll\left|u_{1}\right|,\left|u_{2}\right|$. The results that were obtained while ignoring the terms we do not dispose of, can therefore be seen as a good approximation of the complete results. The time derivative is computed, as for the time derivative in Curle's analogy, in a Lagrangian reference frame to deal with the relatively large time steps between PIV snapshots. 


\section{PIV results}

The mean flow in the cavity is characterized by a large clockwise rotating region in one part of the cavity and a smaller recirculation region in the lower left corner of the cavity(figure 5). The recirculating flow inside the cavity impinges on the separating laminar shear layer, which determines its de-stabilization and triggers the development of vortical structures that convect downstream and then impinge on the aft cavity wall. The shedding of the shear layer is

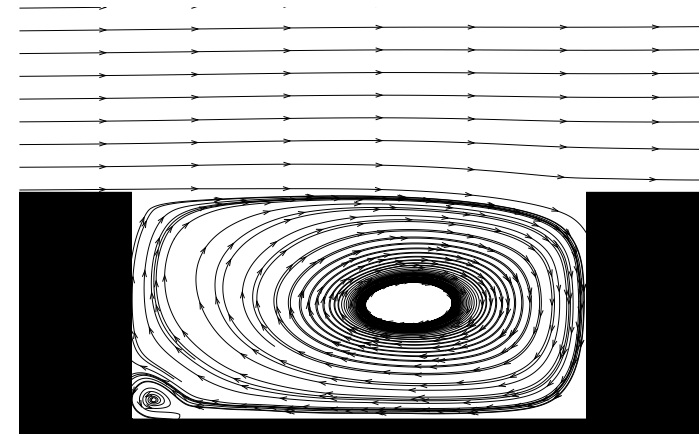

(a) Streamlines

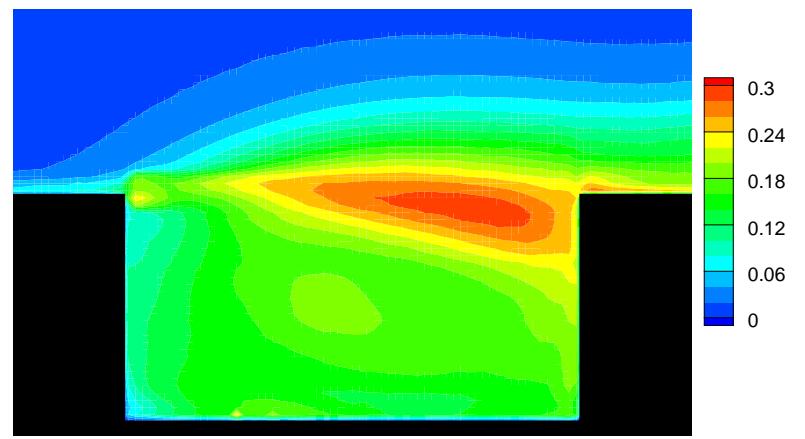

(b) Turbulent intensity normalized to the free stream velocity

Figure 5. (a)Mean flow in the cavity, represented as streamlines. (b) Turbulent intensity.

shown in figure 6, where we give three consecutive snapshots (that also give an indication of the temporal resolution that was achieved). The contour plot in figure 6(a) indicates vorticity. We need to compute the vorticity for the source term in the vortex sound theory (equation (4)). PIV data can be quite noisy if compared to CFD data, and the spatial resolution is also limited. Moreover, adjacent data are obtained from overlapping interrogation windows (by 50\%), so neighboring data are statistically correlated. A standard central difference scheme would then double the vorticity measurement uncertainty with respect to the one computed from an equally noisy but uncorrelated velocity field. ${ }^{22}$ In order to reduce the uncertainty in the measurement of the vorticity field we therefore need to use different techniques. In particular we choose to calculate the vorticity value on every point by computing the circulation around the 8 neighboring points and dividing the result by the enclosed area. This procedures is actually equivalent to computing the vorticity field by applying a central differences scheme to a velocity field that is first smoothed with a $3 \times 1$ points kernel. ${ }^{23}$ The uncertainty in the measurement is reduced when compared to the result obtained by a central difference scheme and the bias due to the overlapping of the interrogation data is negligible since no differences of neighboring points are used in the computation. In the computation of the source term in Curle's analogy a smoothing is automatically implemented by computing the pressure field with an iterative Poisson solver. Figure 6(b) represents the Lamb vector by the Kirchoff vector (flood) with pressure (lines), and figure 6(c) represents their respective time derivatives, i.e. the source terms for the two analogies. It is clear how the source areas fall nicely on top of each other. But, if we look at the root mean square (figure 7) of the terms shown in figure 6(b), in particular where the areas at which the stronger fluctuations occur, we see an important difference between the two methods. For Curle's analogy it looks like the trailing edge of the cavity is the main source of sound (remember that are only the values at the wall here contribute to the emitted sound). For vortex sound theory also the leading edge seems to contribute significantly to the acoustic emission. This result is in contradiction with what is expected from a leading edge. ${ }^{24}$ The acoustic excitation due to vorticity from the cavity interacting with its leading edge is expected to be canceled out by the production of fresh vorticity. Even small values of $(\omega \times \mathbf{u})$ in this area are extremely magnified by the fact that the term $\nabla Y_{1}(\mathbf{y})$ is very large here (and diverges to $\infty$ at the corner itself). Further investigation is therefore needed to fully understand the reasons and the meaning of the presence of these sound sources in our data. 

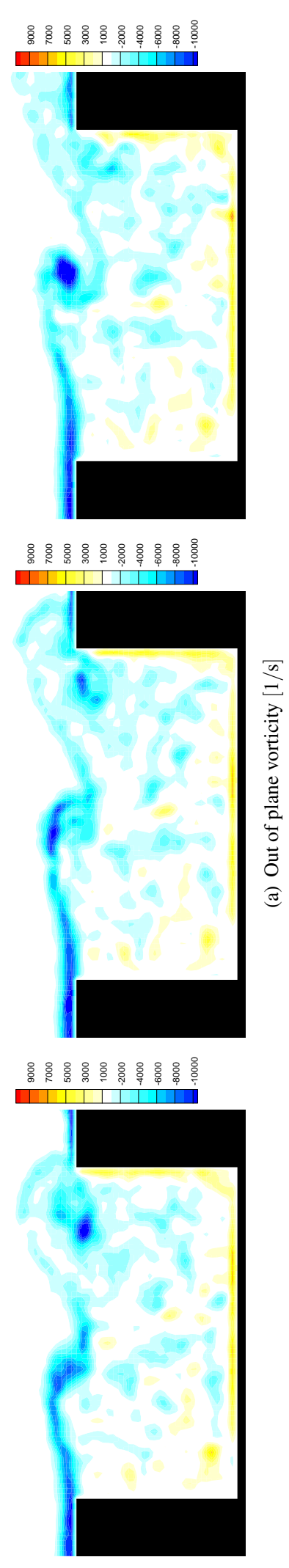
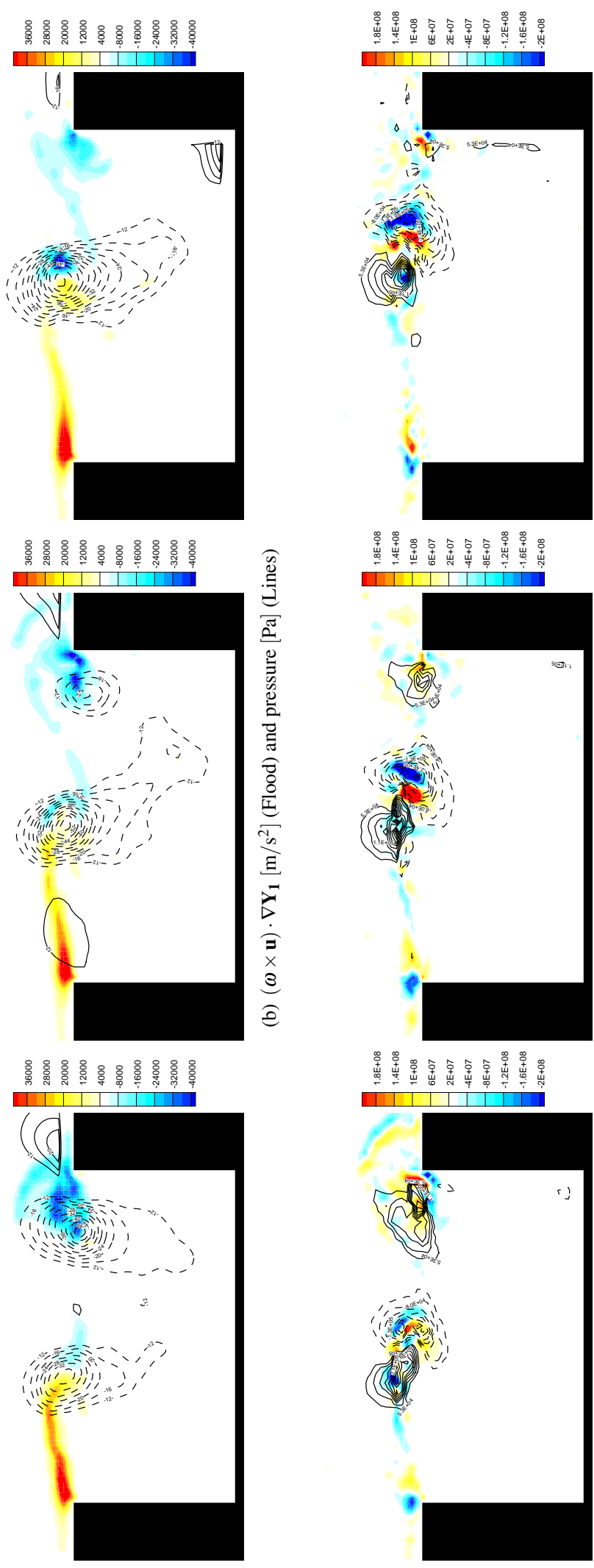

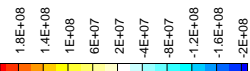

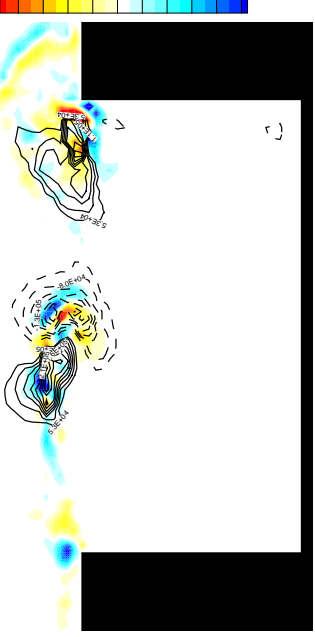

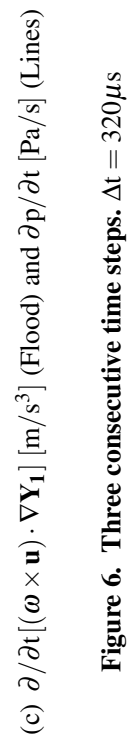




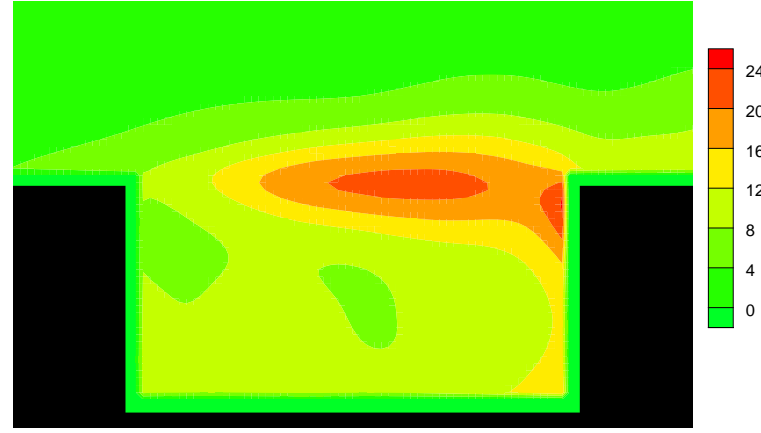

(a) Pressure $[\mathrm{Pa}] \mathrm{rms}$

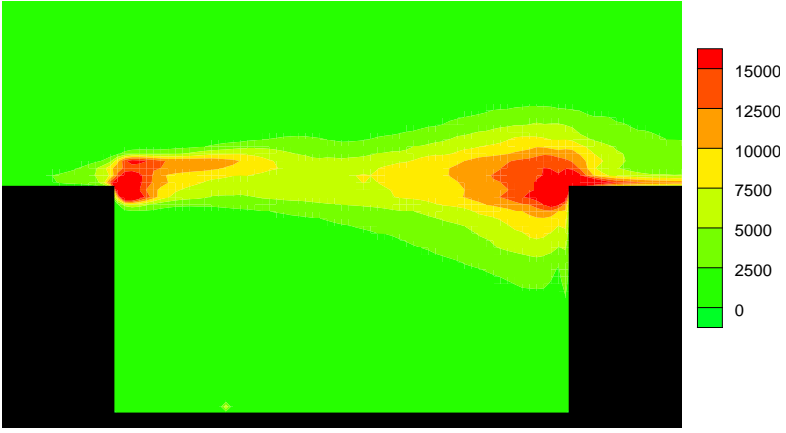

(b) $(\omega \times \mathbf{u}) \cdot \nabla \mathbf{Y}_{\mathbf{1}}\left[\mathrm{m} / \mathrm{s}^{2}\right] \mathrm{rms}$

Figure 7. Root mean square of the source terms for Curle's (a) and vortex sound (b)

\section{Analogies results}

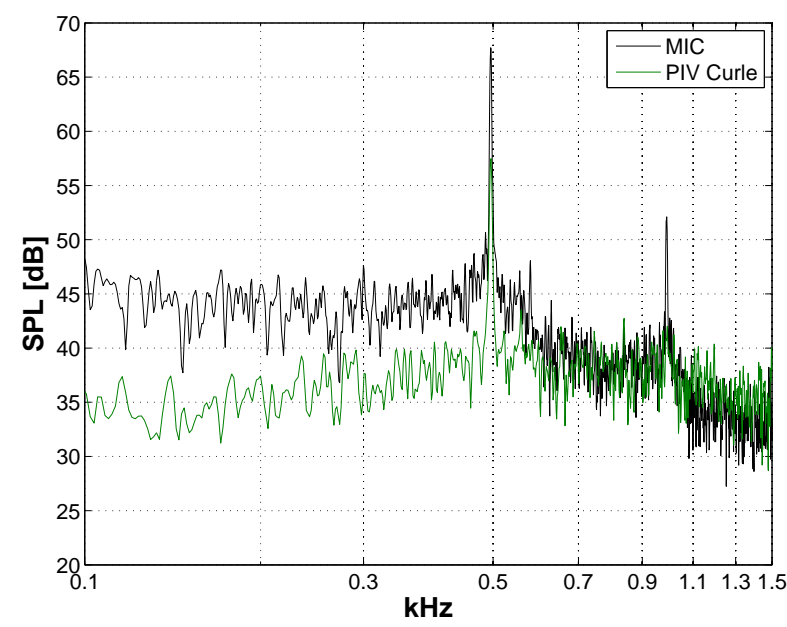

(a) Curle

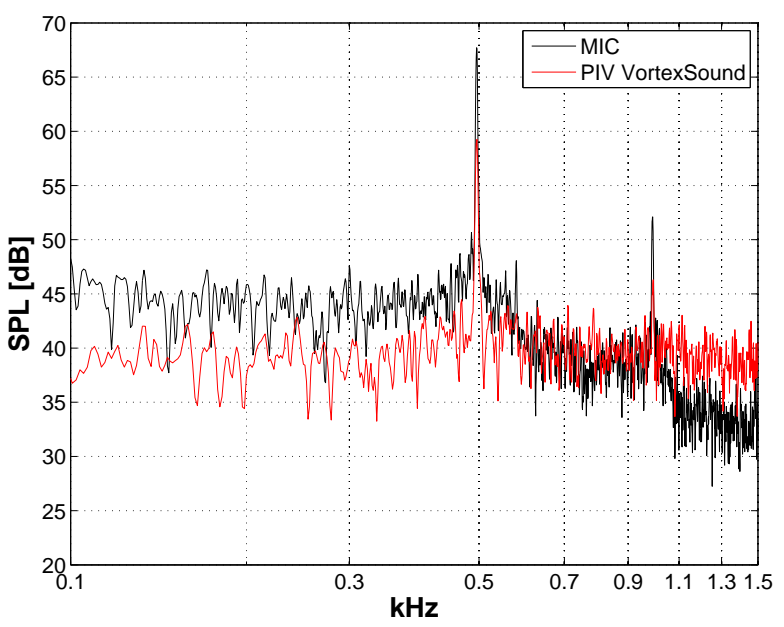

(b) Vortex sound

Figure 8. Power spectra of sound signal from a microphone and of sound computed from PIV at the same locations with the two analogies

Figure 8 shows the power spectra of a microphone positioned 0.5 meters above the cavity (for the position refer to figure 10 and of the acoustic signal computed with Curle's analogy (in green) and vortex sound theory (in red) at the same location). It should be noted that the experimental facility in which we performed the measurements is not acoustically insulated, even though is exceptionally quiet with respect to other similar facilities. Also, due to physical restrictions of our experimental setup, we could not position the microphone at a much larger distance in the far field. The location chosen is approximately one typical wave length ${ }^{\mathrm{b}}$ away from the cavity. The microphone recordings should then be taken just as an indicator and not as a validation data set for our method. Comparing the estimate of the emitted sound obtained by applying the acoustic analogies to the PIV data and the microphone records, we can see that both the main frequency peak and the first harmonic peak are detected by the two approaches. The vortex sound approach seems to better approximate the tonal component of the emitted sound but, at the same time, suffers from a higher broadband noise disturbance in the signal. Both factors are due to the iterative Poisson solver used in the pressure computation, which attenuates the broadband noise but, at the same time, smoothes the tonal component. Table 1 displays the measured overall sound pressure levels at the microphone location. Estimates from Curle's analogy and vortex sound theory are close to each other but are significantly lower than the measured sound pressure level. The main differences occur at the frequencies below that of the main tonal component. In the low-frequency range the microphone measurement includes low-frequency disturbances from the testing equipment (i.e. cooling fans

\footnotetext{
${ }^{\mathrm{b}}$ computed from the main tonal frequency: $\lambda=0.6 \mathrm{~m}$
} 
and especially the pump driving the water cooling system of the laser). In the range of frequencies close to the tonal component we believe that, due to the dimensions of the wind tunnel used in the measurements, standing waves are likely to be present which amplifies the microphone signal in that specific range of frequencies. Are probably the microphones, then, to record higher sound intensities than they should be rather than the analogies prediction to give underestimated results. Further investigation is needed in this respect. Figure 9 shows the power spectra computed

\begin{tabular}{|ccc|}
\hline Mic [dB] & Curle's [dB] & Vortex sound [dB] \\
\hline \hline 76.2 & 70.3 & 72.8 \\
\hline
\end{tabular}

Table 1. Overall sound pressure levels at the microphone position

with the two analogies far enough to be considered in the far field, and the directivity plot at the same distance. The same differences remain as in the microphone location, and the two solutions differ by just a few dB. Figure 10 shows the overall sound pressure level in the region above the cavity (the cavity is centered with respect to the horizontal axis and the position $y=0$ in the vertical direction corresponds to the lead of the cavity). The cavity radiates as a dipole, peaking in the upstream and downstream directions for both the results, and there is little difference between the two solutions.

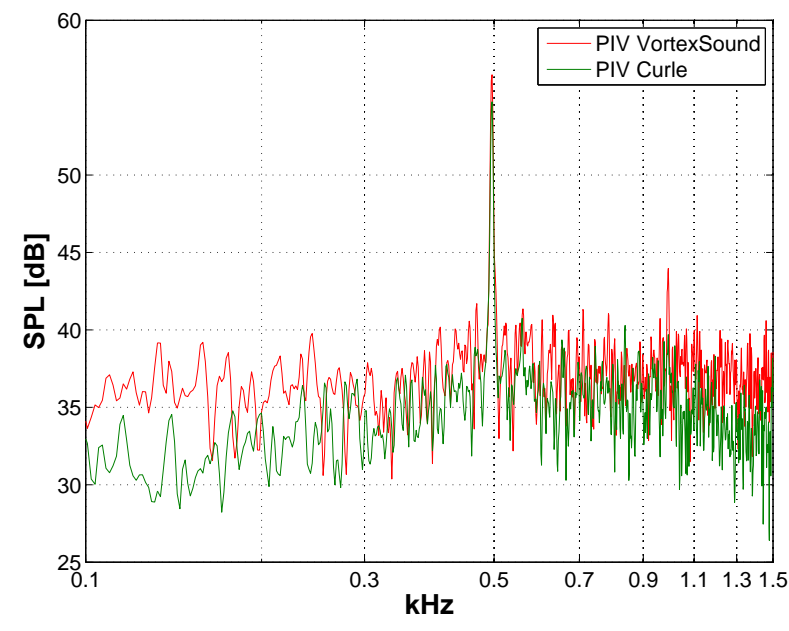

(a) $\mathrm{R}=1.5 \mathrm{~m}, \theta=\pi / 4$

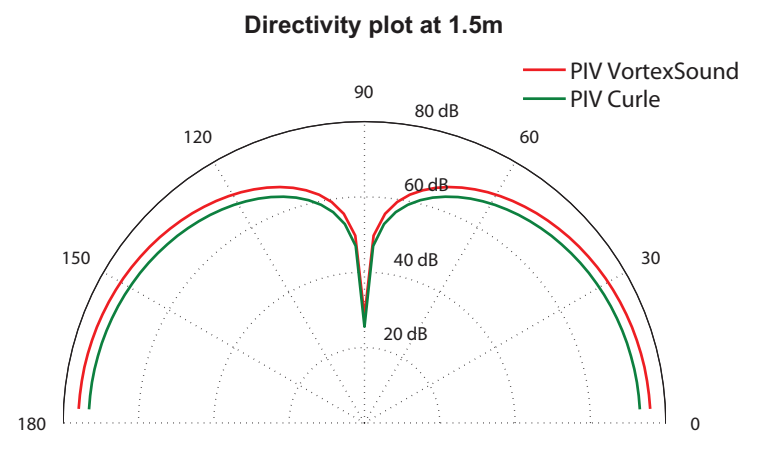

(b) $\mathrm{R}=1.5 \mathrm{~m}$

Figure 9. Power spectra from PIV data in the far field and directivity plot at the same distance

\section{Conclusions}

We compared two different acoustic analogies applied to time-resolved particle image velocimetry (PIV) data. We performed two dimensional, time resolved PIV measurements together with microphone acoustic measurements. Sound is then calculated using Curle's analogy and vortex sound theory and compared with the directly measured acoustic data. The analogies used in the paper are derived under the assumption of low Mach and high Reynolds numbers and for a listener positioned in the far field. We took into account the presence of the non-compact wall in which the cavity is located by using the image principle in the derivation of the solution. An analysis of the source terms for the two analogies reveals that the trailing edge of the cavity is the main source of sound, but unexpectedly, the source term for vortex sound theory shows a strong contribution also from the leading edge. Further investigation is therefore needed to fully understand the reasons and the meaning of the presence of this sound sources in our data. The sound predictions show that both analogies estimate the overall sound pressure level quite well and within a few $\mathrm{dB}$ one to the other. Vortex sound theory seems to provide a better estimate of the amplitude of the tonal component and its harmonics, but it suffers from higher broadband noise compared to the result obtained by Curle's analogy. This is due to the higher smoothing of the data involved in the computation of the source term in Curle's analogy. This 


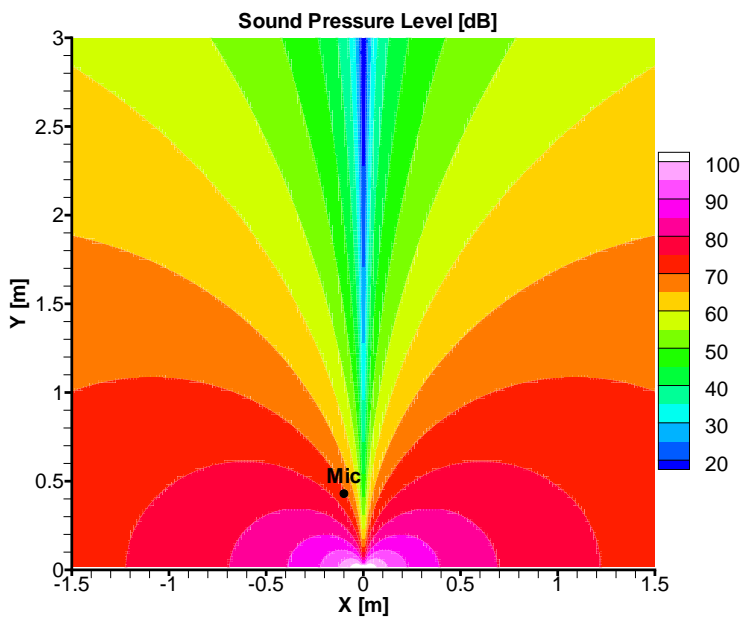

(a) Curle's

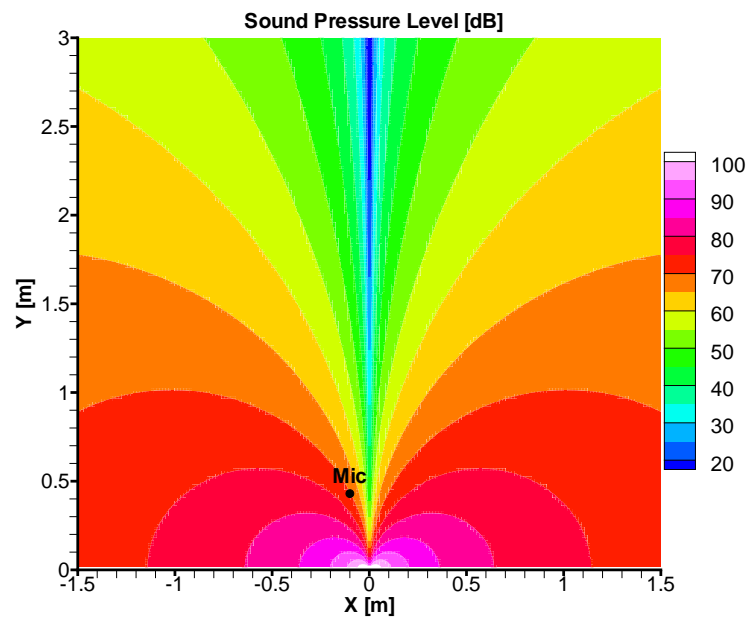

(b) Vortex Sound

Figure 10. Computed overall sound pressure level,[dB]

result makes it clear that great care must be taken in the treatment of the experimental PIV data and especially in the techniques used for spatial and temporal differentiation. The data sets obtained by PIV measurements are in fact quite noisy when compared to uncertainties occurring in CFD results, and the spatial and temporal resolution are still limited. Some conclusions can be drawn about which of the two analogies provides a better prediction of sound emission from PIV data. Curle's analogy has a straightforward formulation that does not depend on the geometry we are considering, as long as the wall normals are correctly defined. Computation of the hydrodynamic pressure from PIV data, on the other hand, is not an easy task especially when its values are needed at the wall. A number of disputable hypotheses and simplifications are required in order to compute a solution. ${ }^{11}$ Moreover, the hydrodynamic pressure, computed through an iterative scheme, becomes heavily smoothed leading to a less sharp acoustic emission. Vortex sound theory needs a Green's function tailored to the body geometry which is not always as straightforward to obtain as in our geometry. The computation of the Lamb vector does not require all the hypotheses and simplifications we needed for the computation of the pressure, but nevertheless attention must be given to the computation of the spatial and temporal derivatives (even more than in Curle's analogy, for which the source term is actually quite smooth) in order to avoid results dominated by noise. Each of the two analogies therefore has its advantages and disadvantages, and which one performs better and is easier to apply depends on individual applications. Further work needs to be done in the differentiation methods used for the computation of the source terms, and a evaluation should be made of the neglected terms in the sound prediction due to the fact that we based the computation on two dimensional measurements.

\section{Acknowledgments}

This work is part of the research program of the Foundation for Fundamental Research on Matter (FOM), which is financially supported by the Netherlands Organization for Scientific Research (NWO).

\section{References}

\footnotetext{
${ }^{1}$ Rowley, W., Colonius, T., and Basu, A., "On self-suistained oscillations in two-dimensional compressible flow over rectangular cavities," $J$. Fluid Mech., Vol. 445, 2001, pp. 315-346.

${ }^{2}$ Ahuja, K. and Mendoza, J., "Effects of cavity dimensions, boundary layer, and temperature on cavity noise with emphasis on benchmark data to validate computational aeroacoustic codes," NASA report CR-4654, 1995.

${ }^{3}$ Gharib, M. and Roshko, A., "The effect of flow oscillations on cavity drag," J. Fluid Mech., Vol. 177, 1987, pp. 501-530.

${ }^{4}$ Block, P., "Noise response of cavities of varying dimensions at subsonic speeds," NASA TN D-8351, 2007.

${ }^{5}$ Tam, C. and Block, P., "On the tones and pressure oscillations induced by flow over rectangular cavities," J. Fluid Mech., Vol. 89, 1978, pp. 373-399.

${ }^{6}$ Grace, S., Dewar, W., and Wroblewski, D., "Experimental investigation of the flow characteristics within a shallow wall cavity for both laminar and turbulent upstream boundary layers," Exp. Fluids, Vol. 36, 2004, pp. 791-804.
} 
${ }^{7}$ Haigermoser, C., Vesely, L., M., N., and Onorato, M., "A time-resolved particle image velocimetry investigation of a cavity flow with a thich incoming boundary layer," Phys. Fluids, Vol. 41, 2008, pp. 227-240.

${ }^{8}$ Haigermoser, C., "Application of an acoustic analogy to PIV data from rectangular cavity flows," Exp. Fluids, Vol. Online first, 2009.

${ }^{9}$ Koschatzky, V., Delfos, R., Boersma, B. J., and Westerweel, J., "Boundary layer influence on cavity noise generation," Advances in Turbulence XII, Proceedings of the 12th EUROMECH European Turbulence Conference, September 7-10, 2009, Marburg, Germany, Vol. $132,2009$.

${ }^{10}$ Koschatzky, V., Boersma, B. J., Scarano, F., and Westerweel, J., "High speed PIV applied to aerodynamic noise investigation," 8th International Symposium on Particle Image Velocimetry-PIV09, 2009.

${ }^{11}$ Koschatzky, V., Moore, P. D., Westerweel, J., Scarano, F., and Boersma, B. J., "High speed PIV applied to aerodynamic noise investigation," Submitted to Exp. Fluids, available on request. [10] is a condensed version of this paper., 2010.

${ }^{12}$ Powell, A., "Theory of Vortex Sound," Journal of the acoustical society of America, Vol. 36, 1964, pp. 177-195.

${ }^{13}$ Howe, M., Theory of vortex sound, Cambridge University Press, 2003.

${ }^{14}$ Westerweel, J., Dabiri, D., and Gharib, M., "The effect of a discrete window offset on the accuracy of cross-correlation analysis of PIV recordings," Exp. Fluids, Vol. 23, 1997, pp. 20-28.

${ }^{15}$ Curle, N., "The influence of solid boundaries upon aerodynamic sound," Proc. Royal Soc. London, Vol. A 231, 1955, pp. 505-514.

${ }^{16}$ Lighthill, M., "On sound generated aerodynamically, Part 1: General theory," Proc. Royal Soc. London, Vol. A 211, 1952, pp. $564-587$.

${ }^{17}$ Powell, A., "Aerodynamic noise and the plane boundary," Journal of the Acoustical Society of America, Vol. 32, 1960, pp. 962990.

${ }^{18} \mathrm{Liu}, \mathrm{X}$. and Katz, J., "Instantaneous pressure and material acceleration measurements using a four-exposure PIV system," Exp. Fluids, Vol. 41, 2006, pp. 227-240.

${ }^{19}$ Moore, P., Lorenzoni, V., and Scarano, F., "Comparison of two techniques for aeroacoustic determination from a rod-airfoil PIV experiment," 8th International Symposium on Particle Image Velocimetry - PIV09, 2009.

${ }^{20}$ Ivanov, V. and Trubetskov, M., Handbook of conformal mapping with computer-aided visualization, CRC Press, 1995.

${ }^{21}$ Abramowitz, M. and Stegun, I., Handbook of mathematical functions, Dover Pubblications, 1965.

${ }^{22}$ Raffel, M.and Willert, C., Wereley, S., and Kompenhans, J., Particle image velocimetry, a practical guide, Springer, 2007.

${ }^{23}$ Westerweel, J., Digital particle image velocimetry: theory and application, Ph.D. thesis, Delft university of technology, mechanical maritime and materials engineering, 1993.

${ }^{24}$ Howe, M., Acoustics of fluid-structure interactions, Cambridge university press, 1998. 